\title{
Editorial
}

\section{Chemical elements in the Universe: Origin and evolution}

The year 1869 is considered as the year of formulation of the Periodic System by Dmitri Mendeleev. The year 2019 was the 150th anniversary of the Periodic Table of Chemical Elements and has therefore been proclaimed the 'International Year of the Periodic Table of Chemical Elements (IYPT 2019)' by the United Nations General Assembly and UNESCO.

An international conference was organized by Indian Institute of Astrophysics (IIA) for four days, starting 16 December 2019, to celebrate the 150 years of the Periodic Table. The objective of the conference titled 'Chemical elements in the Universe: Origin and evolution' was to explore and explain the origin, evolution, and abundance of all the chemical elements in the universe, thus celebrating 150 years of the Periodic Table.

One of the main purposes in organizing this conference was to highlight the work on chemical elements - their abundances and distribution in stars (including Sun), the Milky Way our Galaxy, ISM and other galaxies - done in the past and being pursued now, in particular at IIA as well in other places in India. On a side note, Vainu Bappu, the Founder Director of IIA, was keenly interested in high-resolution spectroscopy and elemental abundances in stars. He worked on elemental abundances in Cepheids among other things. He also designed a high-resolution spectrograph for 1-meter telescope at VBO.

As we know, all the matter around us, and we ourselves, are made up of the elements in the Periodic Table. The origin of these elements by nucleosynthesis happens in different astrophysical environments for example the core of the stars. We seek answers to fundamental questions like: What has been the history of the matter? What observations are required to

This article is part of the Topical Collection: Chemical elements in the Universe: Origin and evolution. determine the composition of this matter in terms of the elements and their isotopes from helium to uranium? Is the history of the matter hidden in the abundance distribution of the observed stellar yields? What is the history of the peculiar and abnormal abundances observed in some stars?

As stated in 'Synthesis of the Elements in Stars', a landmark scientific paper written by Margaret Burbidge, Geoffrey Burbidge, William A. Fowler and Fred Hoyle, published in Reviews of Modern Physics in 1957 , to attempt to understand the sequence of events leading to the formation of the elements it is necessary to study the so-called universal or cosmic abundance curve. Is this abundance curve universal? What is the main source of elements that we observe?

Discussion on solar and solar system abundances, standard cosmic abundances, sites for formation of chemical elements, abundance distributions in disk (thin, thick, ultra-thin) and halo of our Galaxy, nuclear processes for generation of elements, historical developments, etc., was the theme of the conference.

Key questions related to abundance of elements and their inferences in understanding the physical universe starting from Big Bang to the present were explored. Scientists from a range of disciplines deliberated on the origin, evolution, and abundance of all the chemical elements in the universe. The conference was inaugurated by Chief Guest Professor V. S. Ramamurthy, former Secretary, Department of Science $\&$ Technology. Scientist spoke on ways of carrying out studies both in lab and sky, and a public talk by Professor David Lambert, University of Texas at Austin, USA, and Honorary Fellow of IIA, was also held.

The conference also took stock of the progress of understanding in the last 60 years, and highlighted the upcoming spectroscopic surveys and mega facilities that will be instrumental in answering some of these fundamental questions. The research papers/reviews 
presented in the conference are compiled as a special issue of the Journal of Astrophysics and Astronomy titled 'Chemical elements in the Universe: Origin and evolution'. My sincere thanks to all the authors/co- authors for their contributions. Thanks are due to the Journal of Astrophysics and Astronomy chief-editor for providing all the help and support in bringing out this special issue.

Guest Editor

Gajendra Pandey

Indian Institute of Astrophysics, Bengaluru

Email: pandey@iiap.res.in 